\title{
Vehicle recognition analysis in LTE based forward scattering radar
}

\begin{abstract}
By integrating the forward scattering radar (FSR) mode in passive radar can provide many advantages to the conventional passive radar system. The system can benefit from the enhancement in radar cross section (RCS), the low cost and the simple receiver system. In addition, the receiver circuit is less complicated as it does not require a synchronization signal from the transmitter. This paper presents the experimental results for ground target detection and classification in a passive radar system exploiting the effect of forward scatter. The latest 4G Long-Term Evolution (LTE) technology signal is used as the source of the signal transmission. The receiver, the detection and the classification system is explained. Results have shown the system's capability for detecting and classifying ground targets using the FSR technique in passive radar. Hence, it opens up a new frontier in passive radar that can be used for many applications, including border protection, microwave fences, building monitoring and etc.
\end{abstract}

Keyword: LTE; Passive forward scattering radar; Target recognition 